\title{
Curcumin sensitizes glioblastoma to temozolomide by simultaneously generating ROS and disrupting AKT/mTOR signaling
}

\author{
HAITAO YIN $^{1}$, YUN ZHOU ${ }^{1}$, CUIXIA WEN ${ }^{1}$, CHONG ZHOU $^{1}$, WEI ZHANG ${ }^{1}$, \\ XIANG HU ${ }^{1}$, LIFENG WANG ${ }^{2}$, CHUANWEN YOU ${ }^{3}$ and JUNFEI SHAO ${ }^{4}$
}

\begin{abstract}
${ }^{1}$ Department of Radiotherapy, The Central Hospital of Xuzhou, Affiliated Hospital of Southeast University, Xuzhou, Jiangsu;
${ }^{2}$ Department of Oncology, Drum Tower Hospital Affiliated to Medical School of Nanjing University and Clinical Cancer Institute of Nanjing University, Nanjing, Jiangsu; ${ }^{3}$ Department of Oncology, Suqian People's Hospital, Suqian, Jiangsu;

${ }^{4}$ Department of Neurosurgery, Wuxi People's Hospital of Nanjing Medical University, Wuxi, Jiangsu, P.R. China
\end{abstract}

Received May 6, 2014; Accepted July 2, 2014

DOI: $10.3892 /$ or.2014.3342

\begin{abstract}
Temozolomide (TMZ), a DNA alkylating agent, represents the most important chemotherapeutic option for the treatment of glioblastoma in the clinic. Despite its frequent use, the therapeutic efficacy of TMZ remains very limited due to its frequent resistance in glioblastoma. Previous evidence suggested that curcumin (CUM), an ingredient of the Indian spice turmeric, is able to sensitize glioblastoma to TMZ treatment. However, the underlying molecular mechanism remains elusive. In the present study, we performed in vitro and in vivo experiments to evaluate the interaction of CUM and TMZ on the inhibition of glioblastoma and to investigate its potential mechanisms of action using U87MG cell lines and xenograft mouse models. We demonstrated that CUM enhanced the therapeutic response to $\mathrm{TMZ}$ in U87MG glioblastoma by enhancing apoptosis. We then proceeded to investigate the potential apoptotic signaling pathways that are involved. We observed a synergistic effect of the combination of CUM and TMZ in generating reactive oxygen species (ROS) production, suggesting that ROS may contribute to the impact of CUM on sensitizing TMZ treatment. We also showed that CUM and TMZ treatment alone significantly suppressed phosphorylated AKT and mTOR, whereas their combination achieved a more pronounced inhibitory effect. These data indicated that
\end{abstract}

Correspondence to: Dr Lifeng Wang, Department of Oncology, Drum Tower Hospital Affiliated to Medical School of Nanjing University and Clinical Cancer Institute of Nanjing University, 321 Zhongshan Road, Nanjing, Jiangsu 210008, P.R. China

E-mail: lifengwang@nju.edu.cn

Dr Chuanwen You, Department of Oncology, Suqian People's Hospital, Suqian, Jiangsu 223800, P.R. China

E-mail: sqycw@126.com

Key words: curcumin, glioblastoma, temozolomide, sensitization, reactive oxygen species, protein kinase $\mathrm{B} / \mathrm{mammalian}$ target of rapamycin blockage of AKT/mTOR signaling appeared to contribute to the elevated apoptosis caused by the combination treatment with CUM and TMZ. In conclusion, this study provided molecular insights into the effects of CUM on the therapeutic response of glioblastoma to TMZ and opened new avenues for optimizing the therapeutic effects of TMZ-based therapies.

\section{Introduction}

Glioblastoma is one of the most aggressive human tumors with a median survival time of 15 months. Despite considerable advances in cancer treatment in the past decades, glioblastoma remains a highly aggressive disease. Current standard treatment includes a tripartite therapy of surgical resection followed by radiation therapy combined with concurrent administration of the adjuvant DNA alkylating agent temozolomide (TMZ), which represents the most important chemotherapeutic option for glioblastoma treatment (1-3). Novel chemotherapeutic approaches utilizing loco-regional delivery or concurrent chemoradiotherapy have been shown to improve the survival of glioma patients. For instance, the combination of TMZ and radiotherapy has conferred a significant survival advantage compared with radiotherapy alone. In particular, survival benefits of TMZ have been observed in treatments for highgrade gliomas $(4,5)$. Nevertheless, these treatment modalities provide only limited survival benefits, largely due to the frequent development of therapeutic resistance $(6,7)$.

Increasing efforts have been made to understand the molecular basis underlying the occurrence of TMZ-resistance in glioblastoma and to identify combinatorial options to improve the therapeutic efficacy of TMZ. Although the mechanism for resistance development remains unclear, multiple combined modality treatments have been suggested to sensitize TMZ. For example, glioblastoma harboring epidermal growth factor receptor (EGFR) amplification or mutation, EGFR vIII in particular, has been found to be more sensitive to TMZ in combination with cetuximab. In addition, a signal transducer and activator of transcription 3 (STAT3) inhibitor can overcome TMZ resistance in glioblastoma by downregulating 
methylguanine methyltransferase (MGMT) expression (8). It has also been found that a glutathione synthesis inhibitor potentiates the impact of TMZ in glioblastoma (9). Regardless of these advances, an adjuvant therapy for TMZ treatment that benefits a broad range of glioblastoma patients is still needed.

Curcumin (CUM) is a bioactive food component that exhibits a broad spectrum of pharmacological activities including anti-inflammatory, antioxidative and anticancer properties $(10,11)$. While the antioxidative effect of CUM has been well-documented, its anticancer activity has been increasingly studied. CUM is believed to exert anticancer activity in various cancer types by affecting cell proliferation, angiogenesis, invasion, and metastasis via intervention of multiple signaling molecules such as NF- $\mathrm{B}$, PPAR $\gamma$, AMPK and AKT (12-16). Additionally, CUM has also been reported to overcome anti-multidrug-resistance activity (17). Despite its various activities, the molecular basis behind the pharmacological activities of CUM remains largely unknown.

Two previous studies reported that CUM is able to sensitize TMZ in glioblastoma $(18,19)$, suggesting that CUM exhibits the potential to overcome resistance in glioblastoma. In the present study, we validated this finding both in vitro and in vivo and we also investigated the underlying molecular mechanisms of the sensitization of TMZ treatment by CUM. We showed that reactive oxygen species (ROS) generation and AKT-mTOR signaling may be involved in the sensitization of TMZ by CUM in glioblastoma.

\section{Materials and methods}

Cell culture and reagents. Human glioblastoma U87MG cells were obtained from KeyGen Biotech (Nanjing, China). Cells were grown in RPMI-1640 medium supplemented with $10 \%$ fetal bovine serum (both from Gibco, Grand Island, NY, USA) and $1 \%$ penicillin/streptomycin at $37^{\circ} \mathrm{C}$ in a humidified atmosphere with $5 \% \mathrm{CO}_{2}$. TMZ was provided by Schering-Plough (Madison, NJ, USA). PI3K inhibitor LY294002 (purity, 99\%) was obtained from Gibco. Antibodies to mouse PI3K, AKT, phospho-AKT (Thr308), phospho-mTOR (Ser2448), Bad, phospho-Bad (Ser112) and $\beta$-actin were purchased from Cell Signaling (Danvers, MA, USA).

Cell viability assay. Cell viability was measured by a 3-(4,5-dimethylthiazol-2-yl)-2,5-diphenyltetrazolium bromide (MTT) colorimetric assay (Millipore, Billerica, MA, USA) according to the manufacturer's instructions. In brief, $5 \times 10^{3}$ cells/well were plated in 96-well tissue culture plates and grown for $24 \mathrm{~h}$. Cells were then treated with TMZ, CUM alone or their combination at various concentrations. After incubation at $37^{\circ} \mathrm{C}$ for $72 \mathrm{~h}, 10 \mu \mathrm{l}$ of MTT [5 mg/ml dissolved in phosphate-buffered saline (PBS)] was added to each well, and the plates were incubated at $37^{\circ} \mathrm{C}$ for another $4 \mathrm{~h}$. Then, $100 \mu \mathrm{l}$ of isopropanol, $0.1 \mathrm{ml}$ of $0.4 \mathrm{~N} \mathrm{HCl}$, and $50 \mu \mathrm{l}$ of dimethyl sulfoxide were added to solubilize the formazan crystals at room temperature. Within $1 \mathrm{~h}$, the absorbance was measured on a plate reader at a test wavelength of $490 \mathrm{~nm}$. The growth inhibitory rate was determined using the following formula: $($ Inhibition rate, $\%)=\left(1-\mathrm{A}_{\text {treated }} / \mathrm{A}_{\text {control }}\right) \times 100 \%$. The inhibition rate was plotted and the concentration that caused $50 \%$ inhibition $\left(\mathrm{IC}_{50}\right)$ was determined.
ROS detection. Accumulation of intracellular ROS was detected with 2'-7'-dichlorodihydrofluorescein diacetate (DCFH-DA), which crosses cell membranes and is hydrolyzed by intracellular non-specific esterases to non-fluorescent DCFH. In the presence of ROS, DCFH is oxidized to highly fluorescent DCF, which is readily detected by flow cytometry. Cells were seeded into 6 -well plates at a density of $5 \times 10^{5}$ cells/well, cultured overnight, and then incubated with different concentrations of CUM and/or TMZ at $37^{\circ} \mathrm{C}$ for the indicated times. Before harvesting, the cells were incubated with DCFH-DA at a final concentration of $10 \mu \mathrm{M}$ for $20 \mathrm{~min}$. Cells were washed with ice-cold PBS and collected for immediate detection using flow cytometry with excitation at $188 \mathrm{~nm}$ and emission at $525 \mathrm{~nm}$.

Animal studies. Three- to five-week old nu/nu athymic BALB/c mice were purchased from the Shanghai SLAC Laboratory Animal Facility (Shanghai, China). All studies were carried out in compliance with the Institutional Animal Care and Use Committee Guidelines of Nanjing Medical University. Tumors were generated by transplanting $1 \times 10^{6}$ U87MG cells resuspended in PBS (100 $\mu \mathrm{l} /$ mouse) into the right flank. When the tumor volume reached $50-75 \mathrm{~mm}^{3}$, the mice were randomized into 4 groups (6 mice/group): control, CUM-treated group (CUM), TMZ-treated group (TMZ) and CUM plus TMZ-treated group (CUM+TMZ). Mice in the control group were administered vehicle alone, and mice in the treatment groups were administered CUM and/or TMZ at the indicated concentration once daily intraperitoneally (i.p.) for 14 days. Tumor volumes and body weights of the mice were monitored every other day. The sizes of the tumors were measured using microcalipers. The tumor volume (V) was calculated as follows: $\mathrm{V}=\left(\right.$ length $\mathrm{x}$ width $\left.{ }^{2}\right) / 2$. The individual relative tumor volume (RTV) was calculated as follows: $R T V=V_{t} / V_{0}$, where $V_{t}$ is the volume on each day and $V_{0}$ is the volume at the beginning of the treatment. The therapeutic effect of the compounds was expressed as the volume ratio of treatment to control (T/C; ref. 31): T/C $(\%)=($ mean RTV of the treated group/mean RTV of the control group) $\mathrm{x} 100 \%$. On the last day of the experiment, mice were sacrificed and tumor tissues were resected and homogenized in cold RIPA lysis buffer supplemented with protease and phosphatase inhibitors and then processed for immunoblotting.

Apoptosis assay. Cells were treated with TMZ, CUM alone or their combination at various concentrations. After treatment, apoptotic cells were detected by co-staining with Annexin V and propidium iodide (PI), followed by flow cytometry analysis. Cells positive for Annexin V staining are considered to undergo apoptosis. Intratumoral apoptosis was examined by a dUTP nick-end labeling (TUNEL) assay with in situ fluorescein in the sections derived from xenograft tumor tissues using the commercially available fluorescence and colorimetric TUNEL apoptosis assay kit (KeyGen Biotech) following the manufacturer's instructions.

Western blot analysis. Cells or tumor tissues after the indicated treatment were collected and lysed. The supernatant was collected after centrifugation and cell lysates were matched for protein concentration using the Bradford assay. Samples were loaded on SDS-PAGE, transferred to nitrocel- 

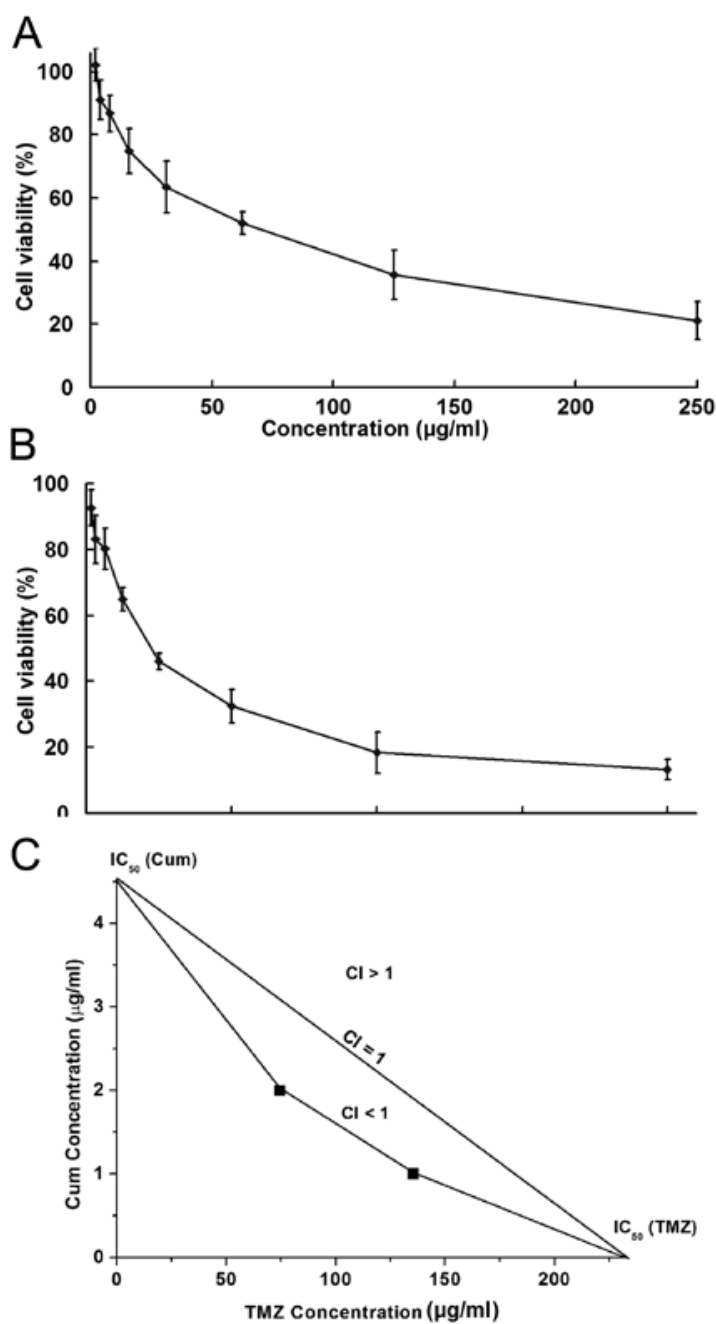

Figure 1. U87MG cells treated with curcumin (CUM) and/or temozolomide (TMZ) at the indicated concentrations for $72 \mathrm{~h}$. Cell viability was measured using an MTT assay. (A) Growth inhibition curve of TMZ; (B) growth inhibition curve of CUM; (C) synergistic analysis of CUM and TMZ by $\mathrm{IC}_{50}$ values. CUM, $1.25 \mu \mathrm{g} / \mathrm{ml}$; TMZ, $15.625 \mu \mathrm{g} / \mathrm{ml}$.

lulose membranes, and blocked in 5\% non-fat milk overnight, followed by an overnight incubation at $4^{\circ} \mathrm{C}$ with primary polyclonal antibodies to mouse PI3K, AKT, phospho-AKT (Thr308), phospho-mTOR (Ser2448), Bad, phospho-Bad (Ser112) and $\beta$-actin. All antibodies were used at a dilution of 1:1,000. Blots were subsequently washed three times with Tris-buffered saline Tween-20 (TBST) and then incubated with the appropriate horseradish peroxidase-conjugated secondary antibodies for $1 \mathrm{~h}$ at room temperature. After three additional TBST washes, the immunoreactive bands were visualized by enhanced chemiluminescence (Amersham Biosciences, Buckinghamshire, UK) according to the manufacturer's instructions. The levels of $\beta$-actin were estimated to check for equal sample loading. Films were scanned and band densities were quantified with densitometric analysis using Scion Image (Epson GT-X700, Tokyo, Japan).

Statistical analysis. All statistical analyses were performed using the Statistical Product and Service Solutions software, version 17.0 (SPSS, Chicago, IL, USA). Data are expressed as means \pm standard deviation (SD). The independent samples
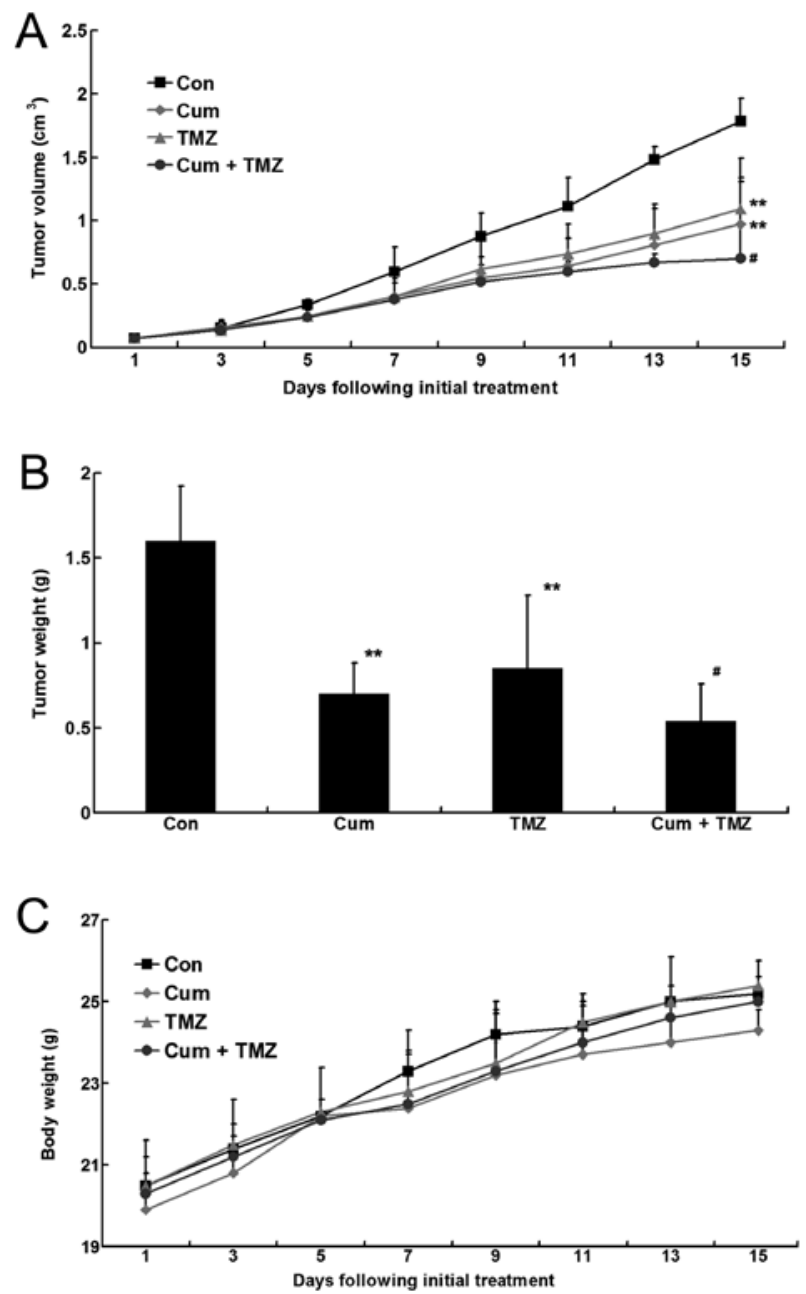

Figure 2. U87MG xenograft mice treated with curcumin (CUM) and/or temozolomide (TMZ) for 14 consecutive days. Body weights and tumor volumes of mice were measured at the indicated time points. (A) Relative tumor volume after the last dose. Data are presented as means $\pm \mathrm{SD} .{ }^{* *} \mathrm{P}<0.05$ vs. the control group; ${ }^{~} \mathrm{P}<0.05$ vs. the group treated with $\mathrm{CUM}$ or TMZ alone; (B) mean tumor weight of each group at the end of the experiment; (C) plot of body weight.

were compared using the Student's t-test. A two-sided P-value of $<0.05$ was considered to indicate a statistically significant difference.

\section{Results}

CUM sensitizes glioblastoma to TMZ treatment. Consistent with previous studies, U87MG cells barely responded to TMZ treatment with an $\mathrm{IC}_{50}$ value of $\sim 390 \mu \mathrm{g} / \mathrm{ml}$ (Fig. 1A). A concentration of $15.63 \mu \mathrm{g} / \mathrm{ml}$, which caused $25.11 \%$ inhibition, was used in the following studies to test the synergistic effect of TMZ in combination with CUM. Similarly, a subtoxic dose $(1.25 \mu \mathrm{g} / \mathrm{ml})$ of CUM was used, which showed an inhibition rate against cell growth of $19.75 \%$ (Fig. 1B). Combination therapy with CUM and TMZ significantly enhanced the effect of TMZ in U87MG cells. A synergistic antitumor effect was clearly observed between CUM and TMZ through the analysis of their $\mathrm{IC}_{50}$ values (Fig. 1C).

In the U87MG xenograft mouse model, CUM and TMZ alone significantly decreased the tumor volume, as well as tumor weight, compared to that in the untreated control group 


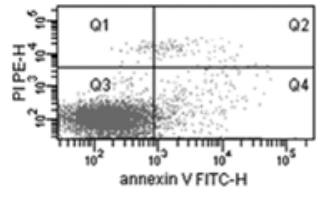

Negative

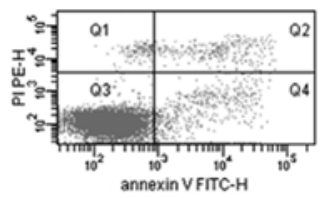

CUM

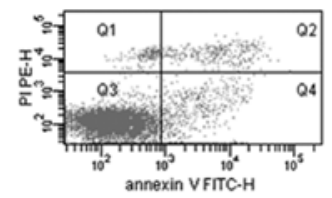

TMZ

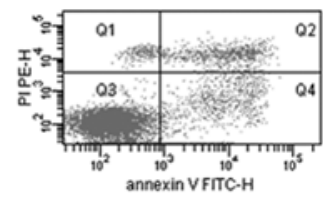

CUM+TMZ

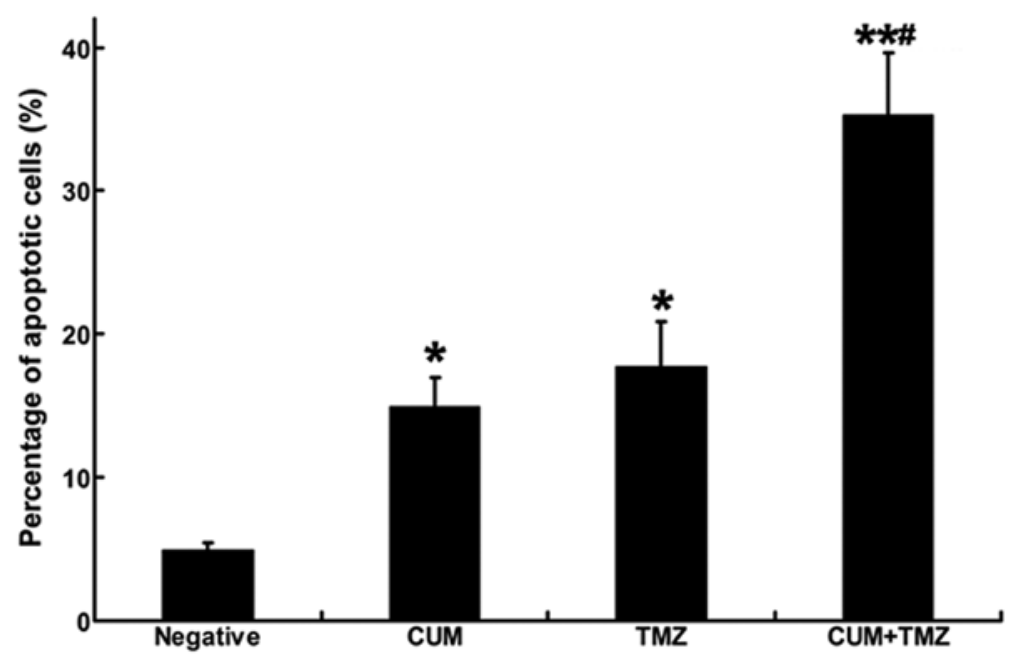

Figure 3. Curcumin (CUM) enhances temozolomide (TMZ)-induced apoptosis in glioblastoma. U87MG cells were treated with CUM (1.25 $\mu \mathrm{g} / \mathrm{ml})$ and/or TMZ $(15.625 \mu \mathrm{g} / \mathrm{ml})$ for $72 \mathrm{~h}$. Upper panel, representative images of flow cytometric analysis of Annexin V/PI dual staining; lower panel, quantification of apoptosis in cells treated with curcumin and/or TMZ. ${ }^{\mathrm{P}}<0.05$ vs. the control group; ${ }^{* *} \mathrm{P}<0.01$ vs. the control group; ${ }^{*} \mathrm{P}<0.05$ vs. the group treated with CUM or TMZ alone.

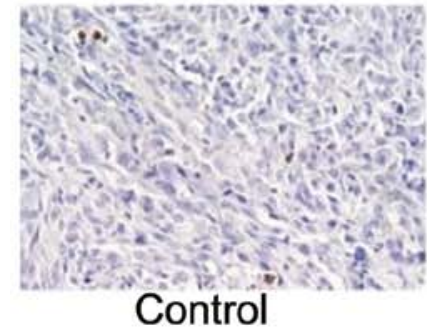

Control

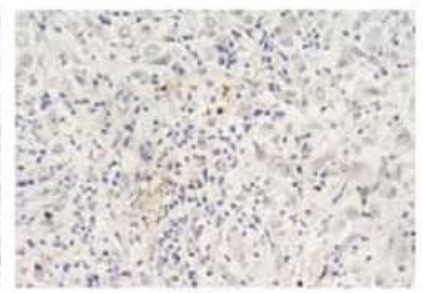

CUM

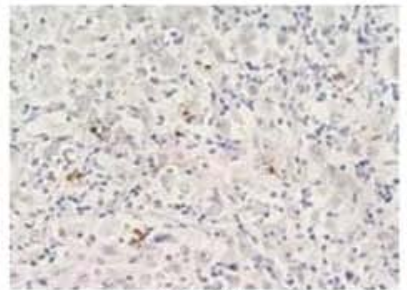

TMZ

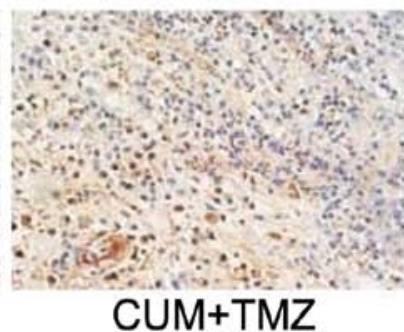

$\mathrm{CUM}+\mathrm{TMZ}$

Figure 4. Curcumin enhances TMZ-induced apoptosis in U87MG xenograft mice. Mice were treated with curcumin (CUM) and/or temozolomide (TMZ) for 14 consecutive days. In situ staining in the TUNEL assay was performed in mouse tumor sections.

$(\mathrm{P}<0.05)$. The combination treatment with CUM and TMZ showed a significantly enhanced effect at inhibiting tumor growth compared with the single treatment (Fig. 2A). A similar trend was observed in the measurement of tumor weight at the end of the experiment (Fig. 2B). However, the mouse body weights remained constant and very similar among the three groups (Fig. 2C).

CUM enhances TMZ-induced apoptosis in glioblastoma. TMZ, as a DNA alkylating agent, has been shown to cause cell growth inhibition primarily via inducing apoptosis in tumor cells (20). We tested whether the increased apoptosis induction mainly accounted for the CUM-enhanced therapeutic efficacy of TMZ. As shown in the upper (early apoptosis) and lower (late apoptosis) right quadrants of Fig. 3, the combination treatment with CUM and TMZ significantly increased the ratio of apoptotic cells compared with either CUM or TMZ treatment alone, suggesting that CUM potentiated the effects of TMZ by sensitizing cells to apoptosis induction. In vivo, intratumoral apoptosis was assessed using a TUNEL assay on the sections derived from xenograft tumor tissues. Apoptotic cells showed brown staining (Fig. 4). Consistent with the results from the in vitro experiment using U87MG cells, intratumoral apoptotic cells were significantly increased in tumor issues from mice treated with the combination of CUM and TMZ compared to TMZ or CUM alone.

CUM and TMZ cause a synergistic effect on ROS generation. Previous studies suggested that TMZ induces apoptosis through a ROS burst (21). Moreover, CUM has been reported to stimulate ROS production during apoptosis, which is induced by anticancer drugs (22). To test whether there is a synergistic effect of CUM and TMZ on ROS production, we measured the ROS amount in U87MG cells after exposure to CUM, TMZ alone or their combination. The single treatment with TMZ significantly generated ROS in U87MG cells, whereas CUM alone only had a non-significant effect. However, ROS production in U87MG cells was markedly increased after 


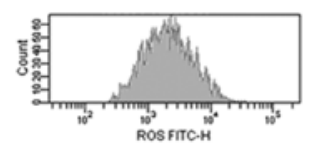

Negative

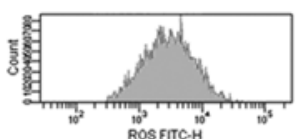

CUM

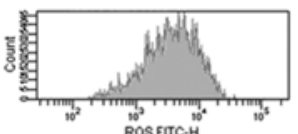

TMZ

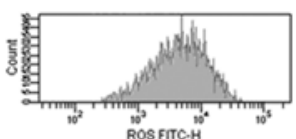

CUM+TMZ

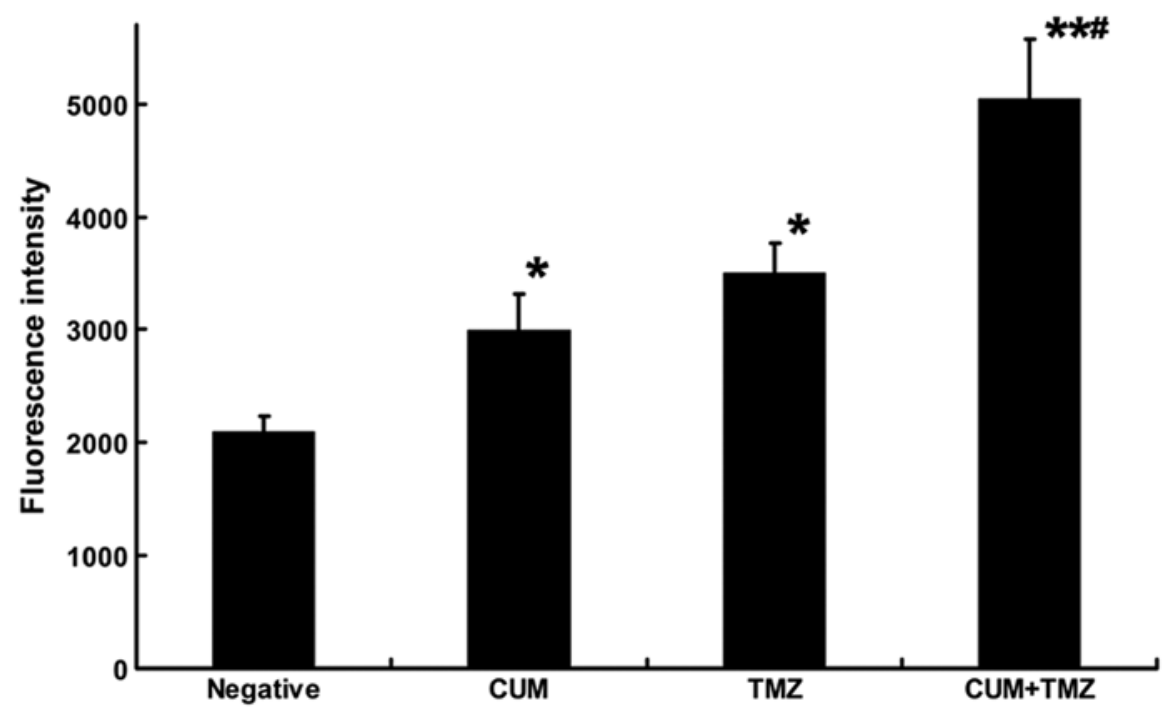

Figure 5. Curcumin (CUM) and temozolomide (TMZ) cause a synergistic effect on ROS generation. Upper panel, representative images of flow cytometric analysis of ROS detection in U87MG cells. Cells were treated with CUM $(1.25 \mu \mathrm{g} / \mathrm{ml})$ and $/$ or TMZ $(15.625 \mu \mathrm{g} / \mathrm{ml})$ for $72 \mathrm{~h}$. Intracellular ROS was detected with DCFH-DA. Lower panel, quantification of ROS production in U87MG cells treated with CUM and/or TMZ. " $\mathrm{P}<0.05$ vs. the control group; ${ }^{* *} \mathrm{P}<0.01 \mathrm{vs.}$ the control group; ${ }^{\sharp} \mathrm{P}<0.05$ vs. the group treated with CUM or TMZ alone.

the combination treatment with CUM and TMZ (Fig. 5), suggesting a synergistic effect of the combination of CUM and TMZ to generate ROS in U87MG cells.

CUM enhances TMZ-disrupted AKT/mTOR signaling. Previous studies have shown that activated AKT signaling overrides TMZ-induced cytotoxicity, leading to a compromised therapeutic effect of TMZ (23). Inhibition of the PI3K/AKT/ mTOR pathway enhances TMZ-induced cytotoxicity (24). In addition, CUM is known to exhibit profound inhibitory effects on the AKT/mTOR signaling network, which accounts for CUM-induced apoptosis in cancer cells $(15,25)$. We examined the AKT/mTOR signaling pathway after CUM and TMZ treatments in U87MG cells. Both CUM and TMZ treatment alone significantly suppressed phosphorylated AKT and mTOR, but their combination achieved a more pronounced inhibitory effect (Fig. 6A). AKT is known to promote cell survival via phosphorylating BAD, a pro-apoptotic member of the Bcl-2 protein family (26). We also observed that the combination treatment of CUM and TMZ significantly decreased the phosphorylation of BAD, compared to the untreated control and treatment with the agents alone (Fig. 6A). Furthermore, using the PI3K inhibitor LY294002, we observed a similar trend of the alterations of phosphorylation of AKT and BAD following CUM and TMZ treatment (Fig. 6A).

These findings were also confirmed in vivo. We observed that the combination treatment of CUM and TMZ significantly decreased phosphorylated AKT expression in the extracts derived from U87MG xenograft tumor tissues, compared to the untreated control and the individual treatments (Fig. 6B). These data suggest that AKT signaling may be implicated in the therapeutic effects of CUM and TMZ in glioblastoma.

\section{Discussion}

In the present study, we showed that CUM is able to sensitize glioblastoma to $\mathrm{TMZ}$ treatment both in vitro and in vivo. We showed that CUM potentiated apoptosis caused by TMZ, and ROS generation may contribute to the impact of CUM when combined with TMZ in glioblastoma cells. We also showed that CUM and TMZ treatment significantly suppressed phosphorylated mTOR, AKT and BAD expression, and their combination achieved a more pronounced inhibitory effect. These data suggest a synergistic effect of the combination of CUM and TMZ in generating ROS and inhibiting AKT/ mTOR signaling in glioblastoma cells.

The mechanism of the development of TMZ resistance has been widely studied. Current insights have mainly focused on the upregulation of DNA repair capacity in human tumors, since TMZ is an alkylating agent prodrug that delivers a methyl group to purine bases of DNA (O6-guanine, N7-guanine and N3-adenine). TMZ-caused DNA lesions are considered to be the key reason to trigger apoptosis and consequently inhibit tumor growth. The primary cytotoxic lesion, O6-methylguanine (O6-MeG), can be removed by MGMT in tumors expressing this protein or tolerated in mismatch repair-deficient tumors (27). Base excision repair (BER), which removes potentially lethal N7- and N3-purine lesions, has also been suggested to contribute significantly to repair or toleration of TMZ treatment. Recently, several small molecule inhibitors of poly(ADP-ribose) polymerase-1 (PARP-1), a critical BER protein, have yielded promising results in combination with TMZ in the clinic.

In the present study, we showed that TMZ combined with CUM significantly increased ROS production and inhibited 

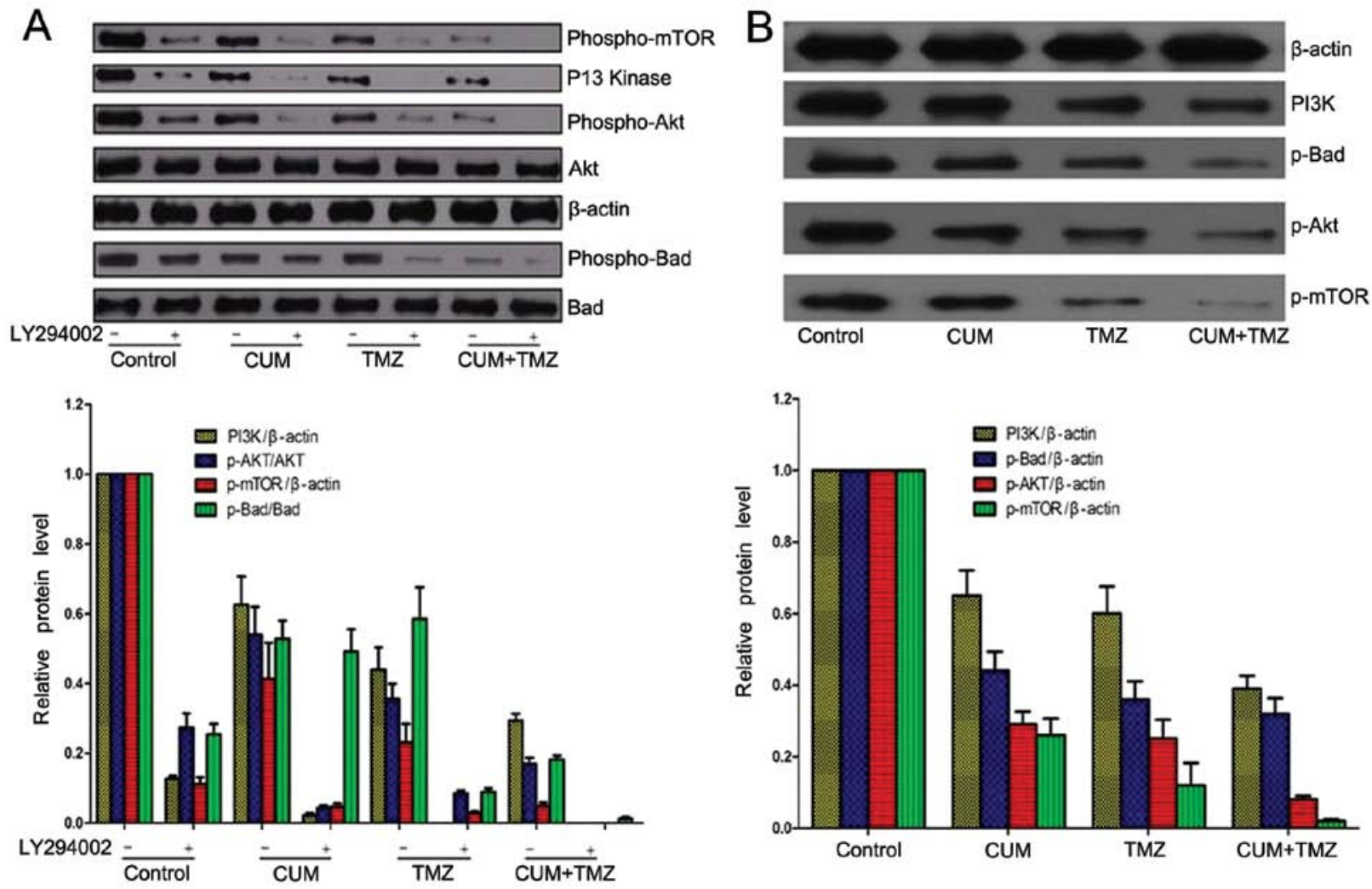

Figure 6. Curcumin (CUM) enhances temozolomide (TMZ)-disrupted AKT/mTOR signaling. (A) CUM enhances TMZ-disrupted AKT/mTOR signaling in U87MG cells. Cells were treated with CUM $(1.25 \mu \mathrm{g} / \mathrm{ml})$ and/or TMZ $(15.625 \mu \mathrm{g} / \mathrm{ml})$ for $72 \mathrm{~h}$ before being subjected to immunoblotting analysis. The upper panel shows representative western blot results, and the lower panel shows densitometric analysis of the relative protein expression. Values are expressed as fold-change compared to the untreated control and are means \pm SEM, $n=3$. (B) CUM enhances TMZ-disrupted AKT/mTOR signaling in U87MG xenograft mice. Mice were treated with CUM and/or TMZ for 14 consecutive days. Tumor tissues were lysed followed by immunoblotting using the indicated antibodies. The upper panel shows representative western blot results, and the lower panel shows densitometric analysis of the relative protein expression. Values are expressed as fold-change compared to the saline-treated control and are means \pm SEM, $n=3$.

AKT/mTOR signaling by decreasing the phosphorylation of AKT, BAD and mTOR in glioblastoma cells. Previous studies have shown that activated AKT signaling overrides TMZ-induced cytotoxicity, leading to a compromised therapeutic effect of TMZ (23). Inhibition of the PI3K/AKT/mTOR pathway has been shown to enhance TMZ-induced cytotoxicity (24). In addition, CUM is known to exhibit profound inhibitory effects on the AKT/mTOR signaling network, which accounts for CUM-induced apoptosis in cancer cells $(15,25)$. Our results are directly or indirectly supported by these previous studies and provide new insight into the mechanisms (such as the AKT/mTOR signaling pathway) of the development of TMZ resistance.

In addition to understanding the mechanism underlying the resistance of TMZ, considerable efforts have been made to identify approaches to overcome this resistance. Although a broad range of pharmacologically active agents, such as inhibitors against PI3K and STAT3, have been suggested to exhibit potential as sensitizers to TMZ, most of these therapeutic options, as an adjuvant therapy to TMZ, are associated with largely enhanced toxicity. In particular, the current standard treatment already includes a tripartite therapy, where both TMZ and radiotherapy are known to cause severe adverse effects in general. The inclusion of extra therapy, particularly one with a considerable impact on vital signaling pathways, will foreseeably be challenged by drug intolerance in patients.
In this respect, CUM, an active ingredient of the dietary spice turmeric, has been consumed for medicinal purposes for a long time (28). CUM modulates various signaling molecules, including inflammatory molecules, transcription factors, enzymes, protein kinases, protein reductases, adhesion molecules, growth factors, receptors, chemokines and even DNA, RNA and metal ions. As a consequence, in the past half century, CUM has been shown to have increasing pharmacological properties including anti-inflammatory, antioxidant, pro-apoptotic, chemopreventive, chemotherapeutic, antiproliferative and wound healing, as well as antinociceptive, antiparasitic and antimalarial properties. However, the current mechanistic insights into the molecular basis underlying the bioactive properties of CUM remain uncertain.

Our findings suggest the important implications of both ROS and AKT-BAD signaling in the therapeutic effect of CUM when combined with TMZ. We acknowledged that the involvement of other pathways is not excluded. For example, our preliminary data suggested that the expression level of the DNA repair gene MGMT, which has been suggested to play an important role in the resistance development of TMZ (29), was modulated by CUM treatment in the xenograft mouse models. To fully understand the biological impact of CUM, more systematic and comprehensive studies are urgently required to elucidate the mechanism of CUM in different scenarios. 
In summary, our data indicated the important implications of apoptosis and AKT-BAD signaling in maintaining the survival of glioblastoma. In addition, ROS generation and the efficient disruption of the AKT-BAD cascade may, at least partially, explain the CUM-sensitized TMZ efficacy in glioblastoma. Although CUM has not yet been approved for the treatment of any human disease, it features an inexpensive, apparently well-tolerated and potentially active adjuvant for TMZ in the treatment of glioblastoma. Our findings provide insights into the molecular basis and will potentially benefit its eventual application in the treatment of glioblastoma.

\section{Acknowledgements}

This study was supported by a grant from the Nanjing Medical Technology Development Project (no. YKK10072), and the Jiangsu Province Health Research Fund (no. H201235).

\section{References}

1. Stupp R, Mason WP, van den Bent MJ, et al: Radiotherapy plus concomitant and adjuvant temozolomide for glioblastoma. N Engl J Med 352: 987-996, 2005.

2. DeAngelis LM: Brain tumors. N Engl J Med 344: 114-123, 2001.

3. Laws ER, Parney IF, Huang W, et al: Survival following surgery and prognostic factors for recently diagnosed malignant glioma: data from the Glioma Outcomes Project. J Neurosurg 99: $467-473,2003$

4. Kapoor R, Revesz T and Powell M: Solitary cervical lymphoma presenting as a neurofibroma. Br J Neurosurg 6 : 583-586, 1992.

5. Perry JR, Bélanger K, Mason WP, et al: Phase II trial of continuous dose-intense temozolomide in recurrent malignant glioma: RESCUE study. J Clin Oncol 28: 2051-2057, 2010.

6. Mirimanoff RO, Gorlia T, Mason W, et al: Radiotherapy and temozolomide for newly diagnosed glioblastoma: recursive partitioning analysis of the EORTC 26981/22981-NCIC CE3 phase III randomized trial. J Clin Oncol 24: 2563-2569, 2006.

7. Mrugala MM and Chamberlain MC: Mechanisms of disease: temozolomide and glioblastoma - look to the future. Nat Clin Pract Oncol 5: 476-486, 2008

8. Kohsaka S, Wang L, Yachi K, et al: STAT3 inhibition overcomes temozolomide resistance in glioblastoma by downregulating MGMT expression. Mol Cancer Ther 11: 1289-1299, 2012.

9. Kohsaka S, Takahashi K, Wang L, et al: Inhibition of GSH synthesis potentiates temozolomide-induced bystander effect in glioblastoma. Cancer Lett 331: 68-75, 2013.

10. Joe B, Vijaykumar M and Lokesh BR: Biological properties of curcumin-cellular and molecular mechanisms of action. Crit Rev Food Sci Nutr 44: 97-111, 2004.

11. Maheshwari RK, Singh AK, Gaddipati J and Srimal RC: Multiple biological activities of curcumin: a short review. Life Sci 78 2081-2087, 2006.

12. Kunnumakkara AB, Anand P and Aggarwal BB: Curcumin inhibits proliferation, invasion, angiogenesis and metastasis of different cancers through interaction with multiple cell signaling proteins. Cancer Lett 269: 199-225, 2008.
13. Das T, Sa G, Saha B and Das K: Multifocal signal modulation therapy of cancer: ancient weapon, modern targets. Mol Cell Biochem 336: 85-95, 2010.

14. Basile V, Ferrari E, Lazzari S, Belluti S, Pignedoli F and Imbriano C: Curcumin derivatives: molecular basis of their anticancer activity. Biochem Pharmacol 78: 1305-1315, 2009.

15. Hussain AR, Al-Rasheed M, Manogaran PS, et al: Curcumin induces apoptosis via inhibition of PI3'-kinase/AKT pathway in acute T cell leukemias. Apoptosis 11: 245-254, 2006.

16. Zheng S and Chen A: Activation of PPARgamma is required for curcumin to induce apoptosis and to inhibit the expression of extracellular matrix genes in hepatic stellate cells in vitro. Biochem J 384: 149-157, 2004.

17. Lu JJ, Cai YJ and Ding J: The short-time treatment with curcumin sufficiently decreases cell viability, induces apoptosis and copper enhances these effects in multidrug-resistant K562/A02 cells. Mol Cell Biochem 360: 253-260, 2012.

18. Ramachandran C, Nair SM, Escalon E and Melnick SJ: Potentiation of etoposide and temozolomide cytotoxicity by curcumin and turmeric force in brain tumor cell lines. J Complement Integr Med 9: Article 20,2012.

19. Chen CC, Taniguchi T and D'Andrea A: The Fanconi anemia (FA) pathway confers glioma resistance to DNA alkylating agents. J Mol Med 85: 497-509, 2007.

20. Roos WP, Batista LF, Naumann SC, et al: Apoptosis in malignant glioma cells triggered by the temozolomide-induced DNA lesion $O^{6}$-methylguanine. Oncogene 26: 186-197, 2007.

21. Lin CJ, Lee CC, Shih YL, et al: Resveratrol enhances the therapeutic effect of temozolomide against malignant glioma in vitro and in vivo by inhibiting autophagy. Free Radic Biol Med 52: 377-391, 2012.

22. Sánchez Y, Simón GP, Calviño E, de Blas E and Aller P: Curcumin stimulates reactive oxygen species production and potentiates apoptosis induction by the antitumor drugs arsenic trioxide and lonidamine in human myeloid leukemia cell lines. J Pharmacol Exp Ther 335: 114-123, 2010.

23. Hirose Y, Katayama M, Mirzoeva OK, Berger MS and Pieper RO: Akt activation suppresses Chk2-mediated, methylating agentinduced $\mathrm{G}_{2}$ arrest and protects from temozolomide-induced mitotic catastrophe and cellular senescence. Cancer Res 65 : 4861-4869, 2005.

24. Chen L, Han L, Shi Z, et al: LY294002 enhances cytotoxicity of temozolomide in glioma by down-regulation of the PI3K/Akt pathway. Mol Med Rep 5: 575-579, 2012.

25. Yu S, Shen G, Khor TO, Kim JH and Kong AN: Curcumin inhibits Akt/mammalian target of rapamycin signaling through protein phosphatase-dependent mechanism. Mol Cancer Ther 7: 2609-2620, 2008.

26. Datta SR, Dudek H, Tao X, et al: Akt phosphorylation of BAD couples survival signals to the cell-intrinsic death machinery. Cell 91: 231-241, 1997.

27. Zhang J, Stevens MF and Bradshaw TD: Temozolomide: mechanisms of action, repair and resistance. Curr Mol Pharmacol 5: 102-114, 2012.

28. Gupta SC, Patchva S, Koh W and Aggarwal BB: Discovery of curcumin, a component of golden spice, and its miraculous biological activities. Clin Exp Pharmacol Physiol 39: 283-299, 2012.

29. Hegi ME, Diserens AC, Gorlia T, et al: $M G M T$ gene silencing and benefit from temozolomide in glioblastoma. N Engl J Med 352: 997-1003, 2005. 